\title{
IMPORTANCIA DEL JAGÜEY GANADERO EN LA CONSERVACIÓN LOCAL DE AVES SILVESTRES EN EL CARIBE COLOMBIANO
}

\section{CATTLE POND IMPORTANCE IN THE LOCAL CONSERVATION OF WILD BIRDS IN THE COLOMBIAN CARIBBEAN}

\author{
Jaime De La Ossa-V. ${ }^{1}$, Silvia Galván-Guevara², Alejandro De La Ossa-Lacayo³
}

1 Universidad de Sucre, Facultad de Ciencias Agropecuarias. Grupo Investigación Biodiversidad Tropical, Colombia. jaimedelaossa@yahoo.com. 2 Universidad de Cartagena, Maestría en Ciencias Ambientales. Grupo de Investigación en Biodiversidad Tropical de la Universidad de Sucre, Colombia. ${ }^{3}$ Universidad Pontificia Javeriana de Colombia, Maestría en Desarrollo Rural, Colombia.

Rev. U.D.C.A Act. \& Div. Cient. 15(1): 181 - 186, 2012

\section{RESUMEN}

Se llevó a cabo un inventario de la avifauna residente o visitante asociada a un jagüey o laguna artificial de la costa Caribe de Colombia, que se utiliza como reserva de agua para el ganado vacuno en la época seca y que también sirve como fuente de agua para las comunidades cercanas. Se hizo el registro de individuos de cada especie durante la época seca e inicio de la época de lluvias, mediante el método de observación constante, por periodos de tiempo preestablecidos, usando dos puntos fijos de conteo. Se determinó una comunidad de aves que mostró 28 especies pertenecientes a 15 familias, de las cuales, se identificaron como residentes continuos (RC), 11 especies (39,3\%), como visitantes regulares (VR), 7 especies (25\%) y como visitantes ocasionales (VO), 10 especies (35,7\%), lo que permite aceptar la importancia estratégica de este tipo de lagunas en la conservación de la biodiversidad local, mostrando que los agroecosistemas pueden conservar una sustancial parte de la biodiversidad de su anterior ecosistema natural.

Palabras clave: Jagüey, avifauna, abundancia, Caribe, Colombia.

\section{SUMMARY}

Was carried out an inventory of the resident or visitor avifauna associated to an artificial pond or "jagüey" of the Colombian
Caribbean coast that is used as cattle water reservation for the dry season and that it is also used as water source of water for its near human communities. The registration of individuals of each species was made during the dry season and at the beginning of the rainy season, using two fixes observation points. In this bird community, 28 species belonging to 15 families were determined, of which as continuous residents (RC) 11 species (39,3\%), as regular visitors (VR) 7 species (25\%), and as occasional visitors (VO) 10 species (35.7\%) were identified; this allows to accept the strategic importance of this type of artificial lagoons in the conservation of the local biodiversity, showing that the agroecosystems can conserve an important part of the biodiversity of its previous natural ecosystem.

Key words: Artificial pond, avifauna, abundance, Caribbean, Colombia.

\section{INTRODUCCIÓN}

La avifauna posee un gran valor para la diversidad biológica nacional y las aves silvestres tienen intrínsecos valores para la sociedad (Díaz \& Armesto, 2003). Dado este reconocimiento es fundamental que se lleven a cabo inventarios de las poblaciones existentes, seguimiento y control de los cambios poblacionales, en función del tiempo y de las condiciones ambientales, ya que existe el riesgo de declinación poblacional por factores diversos, pero significativamente de origen 
humano: como pérdida de hábitat, introducción de especies exóticas, comercio de mascotas, toxicidad ambiental, producción agrícola y predación humana (Canavelli et al. 2004), a los cuales, no escapa la región Caribe colombiana, dedicada, principalmente, a labores agrícolas, como parte fundamental de su economía, siendo la ganadería de carne una de las actividades de mayor impacto negativo, ambientalmente hablando (Mahecha et al. 2002).

Ahora, lugares comparativamente pequeños bien protegidos, como lagunas artificiales, cercas vivas o parches de bosques, pueden adquirir gran importancia como hábitat, porque permiten la concentración de la avifauna local (Salinas et al. 2007). Para Centroamérica, se reconocen prácticas silvopastoriles, por medio de las cuales, se ha logrado aumentar la producción animal, recuperar áreas degradadas que, a su vez, ayudan a ofrecer refugio permanente u ocasional a muchas poblaciones de avifauna; se encontró que las cercas vivas, por ejemplo, son de gran importancia para las aves residentes, especialmente, en la época seca, cuando los árboles están en floración y atraen a muchos polinizadores que pueden servirles de alimento (Sáenz \& Menacho, 2005), lo que concuerda para los jagüeyes en lo que a fauna silvestre hace referencia (Botero et al. 2009).

También es claro que en ambientes acuáticos construidos para fines ganaderos, algunas especies de aves pueden sobrevivir (Botero et al. 2009), por ejemplo, entre las aves acuáticas, algunas especies de ellas migratorias, hallan refugio temporal en estos cuerpos de agua, mientras otras, no migratorias, se tornan residentes permanentes (Botero et al. 2009) y en estos lugares es factible hallar casi todas las especies comunes para el área (Fajardo et al. 2008). Entonces, ante los impactos antrópicos, cada vez más evidentes por sus consecuencias, los jagüeyes se convierten en una importante alternativa de conservación biológica (Declerck et al. 2006) y en donde es factible hacer listados, censos y estudios de abundancia poblacional, que conduzcan a posteriores planes de manejo, con fines conservacionistas.

En el presente trabajo, se llevó a cabo observación, identificación y determinación de abundancia relativa de las aves que habitan un jagüey, en la zona de sabanas antrópicas del departamento de Sucre, área representativa de la zona seca del Caribe colombiano. Se compara la presencia ausencia de las diferentes poblaciones de acuerdo con la estación pluviométrica.

\section{MATERIALES Y MÉTODOS}

Área de estudio: Granja el Perico, Facultad de Ciencias Agropecuarias de la Universidad de Sucre, Colombia, localizada a $9^{\circ} 12^{\prime} 45.1^{\prime \prime}$ ' N y $75^{\circ} 24^{\prime \prime} 1.8^{\prime \prime \prime} \mathrm{O}$, a 146 m.s.n.m.
El área de estudio posee en espejo de agua de 0,9ha en su máxima cota de inundación. La zona, se clasifica como perteneciente al bosque seco tropical, caracterizada como un ecosistema de piso térmico cálido, con periodos de lluvias anuales bien definidos (Botero et al. 2009), incluye grandes extensiones de sabanas antrópicas y no está conectada a un río ni pertenece a una cuenca permanente; su dinámica está determinada por el flujo de las escorrentías. Específicamente, el jagüey estudiado presenta en sus orillas abundante vegetación, con una cobertura del $60 \%$ y un dosel medio de $15 \mathrm{~m}$, así como una densa población flotante de Pistia stratiotes (oreja de mulo) (De La Ossa et al. 2009).

Métodos: Se hizo un inventario de las aves presentes y se registró el número de individuos de cada especie, durante la época seca (febrero-abril) e inicio de la época de lluvias (mayo-julio), de 2009, según lo propuesto por Dunn \& Ralph (2004), mediante el método de observación constante por periodos de tiempo preestablecidos; se usó el punto fijo de conteo (Farnsworth et al. 2002); se trabajó tres veces a la semana, entre las 06:00 y las 10:00 horas y entre las 16:00 y las 19:00 horas, con dos puntos fijos de avistamiento. La identificación taxonómica se llevó a cabo in situ (Remsen et al. 2008).

Análisis de los datos: Mediante el programa InfoStat 2011e-free, se verificó el supuesto de normalidad de los datos y homogeneidad de varianza, se aplicó ANOVA y prueba de múltiples rangos (diferencias mínimas significativas); se establecieron cálculos de abundancia relativa en porcentaje, con las siguientes categorías: residente continuo (RC) (especies que habitan exclusivamente el lugar), visitante regular (VR) (permanecen parte del día en el jagüey) y visitante ocasional (VO) (especies que llegan a forrajear o beber agua y se van de inmediato) (Hernández-Vásquez, 2000).

\section{RESULTADOS Y DISCUSIÓN}

En la tabla 1, se presentan los resultados obtenidos durante los seis meses de estudio. Se registraron 28 especies pertenecientes a 15 familias, de las cuales, se identificaron como residentes continuos (RC), 11 especies (39,3\%); como visitantes regulares (VR), 7 especies (25\%) y como visitantes ocasionales (VO), 10 especies (35,7\%). Igualmente, se presentan los valores de abundancia relativa en porcentaje para cada especie por periodo analizado.

No se encontraron diferencias significativas al aplicar ANOVA para comparar los meses de estudio, pero sí se hallaron para el carácter (Tabla 2). Al hacer un análisis de diferencias mínimas significativas para el carácter ocupacional por especie, significativamente se diferencian las especies 
Tabla 1. Registro y valores de abundancia de las aves asociadas a un jagüey de la costa Caribe colombiana ((F) febrero, $(M)$ marzo, (A) abril), ((My) mayo, (J) junio, (Jl) julio), (N.C) Nombre común, (C) Carácter, (RC) Residente continuo, (VR) Visitante regular, (VO) Visitante ocasional, $(\bar{X})$ Media por periodo estudiado, (\%) Representación porcentual por periodo.

\begin{tabular}{|c|c|c|c|c|c|c|c|c|c|c|c|c|c|}
\hline \multirow{2}{*}{ Familia } & \multirow{2}{*}{ Especie } & \multirow{2}{*}{ N.C } & \multirow{2}{*}{ C } & \multicolumn{5}{|c|}{ Sequía } & \multicolumn{5}{|c|}{ Lluvias } \\
\hline & & & & $\mathrm{F}$ & M & A & $\bar{X}$ & $\%$ & My & $\mathrm{J}$ & $\mathrm{Jl}$ & $\bar{X}$ & $\%$ \\
\hline \multirow{5}{*}{ ARDEIDAE } & Butorides striatus & Garcipolo & VO & 1 & 0 & 0 & 0,33 & 0,38 & 1 & 2 & 1 & 1,33 & 1,85 \\
\hline & Bubulcus ibis & Garza del ganado & $\mathrm{RC}$ & 3 & 5 & 4 & 4,00 & 4,55 & 2 & 5 & 3 & 3,33 & 4,63 \\
\hline & Ardea alba & Garza real & VO & 1 & 1 & 1 & 1,00 & 1,14 & 0 & 0 & 0 & 0,00 & 0,00 \\
\hline & $\begin{array}{l}\text { Nycticorax } \\
\text { nycticorax }\end{array}$ & Guaco & $\mathrm{RC}$ & 1 & 1 & 1 & 1,00 & 1,14 & 1 & 1 & 2 & 1,33 & 1,85 \\
\hline & Tigrisoma lineatum & Vaco tigre & $\mathrm{RC}$ & 1 & 1 & 0 & 0,67 & 0,76 & 0 & 2 & 1 & 1,00 & 1,39 \\
\hline \multirow{2}{*}{ CATHARTIDAE } & Cathartes aura & Laura & VR & 2 & 3 & 1 & 2,00 & 2,27 & 1 & 0 & 0 & 0,33 & 0,46 \\
\hline & Coragyps atratus & Golero & VR & 2 & 3 & 1 & 2,00 & 2,27 & 1 & 2 & 4 & 2,33 & 3,24 \\
\hline \multirow{2}{*}{ ACCIPITRIDAE } & Busarellus nigricollis & Gavilán & VR & 1 & 1 & 0 & 0,67 & 0,76 & 0 & 1 & 1 & 0,67 & 0,93 \\
\hline & Buteo magnirostris & Gavilán & VR & 2 & 2 & 2 & 2,00 & 2,27 & 2 & 2 & 2 & 2,00 & 2,78 \\
\hline \multirow{2}{*}{ FALCONIDAE } & Milvago chimachima & Pigua & VR & 3 & 5 & 4 & 4,00 & 4,55 & 3 & 4 & 5 & 4,00 & 5,56 \\
\hline & Caracara plancus & Caracara & VR & 0 & 0 & 1 & 0,33 & 0,38 & 0 & 0 & 0 & 0,00 & 0,00 \\
\hline JACANIDAE & Jacana jacana & Gallito de ciénaga & $\mathrm{RC}$ & 7 & 8 & 8 & 7,67 & 8,71 & 6 & 9 & 8 & 7,67 & 10,65 \\
\hline \multirow{2}{*}{ COLUMBIDAE } & $\begin{array}{l}\text { Columba } \\
\text { cayennensis }\end{array}$ & Guarumera & VO & 0 & 0 & 0 & 0,00 & 0,00 & 1 & 0 & 1 & 0,67 & 0,93 \\
\hline & Columbina talpacoti & Tierrerita & VO & 9 & 9 & 11 & 9,67 & 10,98 & 7 & 5 & 4 & 5,33 & 7,41 \\
\hline \multirow{2}{*}{ PSITTACIDAE } & Aratinga pertinax & Cotorra & VO & 3 & 4 & 4 & 3,67 & 4,17 & 5 & 4 & 5 & 4,67 & 6,48 \\
\hline & Brotogeris jugularis & Periquito & VO & 6 & 6 & 5 & 5,67 & 6,44 & 2 & 1 & 0 & 1,00 & 1,39 \\
\hline \multirow{2}{*}{ CUCULIDAE } & Crotophaga ani & Cocinera & $\mathrm{RC}$ & 11 & 10 & 15 & 12,00 & 13,64 & 11 & 14 & 10 & 11,67 & 16,20 \\
\hline & Crotophaga major & Cocinera & $\mathrm{RC}$ & 3 & 2 & 4 & 3,00 & 3,41 & 1 & 3 & 3 & 2,33 & 3,24 \\
\hline ALCENIDAE & Ceryle torquata & Martin Pescador & VR & 0 & 0 & 1 & 0,33 & 0,38 & 1 & 0 & 0 & 0,33 & 0,46 \\
\hline PICIDAE & \begin{tabular}{|l} 
Melanerpes \\
rubricapillus
\end{tabular} & Carpintero & VO & 0 & 1 & 0 & 0,33 & 0,38 & 1 & 0 & 0 & 0,33 & 0,46 \\
\hline \multirow{3}{*}{ TYRANNIDAE } & $\begin{array}{l}\text { Arundinicola } \\
\text { leucocephala }\end{array}$ & Niderito & $\mathrm{RC}$ & 2 & 2 & 1 & 1,67 & 1,89 & 2 & 2 & 2 & 2,00 & 2,78 \\
\hline & Pitangus sulphuratus & Chichafria & $\mathrm{RC}$ & 6 & 7 & 4 & 5,67 & 6,44 & 4 & 5 & 7 & 5,33 & 7,41 \\
\hline & $\begin{array}{l}\text { Tyrannus } \\
\text { melancholicus }\end{array}$ & Siriri & $\mathrm{RC}$ & 3 & 2 & 5 & 3,33 & 3,79 & 2 & 4 & 1 & 2,33 & 3,24 \\
\hline HIRUNDINIDAE & Progne tapera & Golondrina & VO & 5 & 6 & 8 & 6,33 & 7,20 & 0 & 0 & 0 & 0,00 & 0,00 \\
\hline TROGLODYTIDAE & $\begin{array}{l}\text { Campylurhyncus } \\
\text { griseus }\end{array}$ & Chupa huevo & $\mathrm{RC}$ & 6 & 8 & 5 & 6,33 & 7,20 & 10 & 9 & 8 & 9,00 & 12,50 \\
\hline THRAUPIDAE & Thraupis episcopus & Azulejo & $\mathrm{RC}$ & 3 & 3 & 2 & 2,67 & 3,03 & 1 & 4 & 3 & 2,67 & 3,70 \\
\hline \multirow{2}{*}{ FRINGILLIDAE } & Saltator coerulescens & Saltarín & VO & 0 & 1 & 1 & 0,67 & 0,76 & 0 & 0 & 0 & 0,00 & 0,00 \\
\hline & Sicalis flaveola & Canario & VO & 3 & 0 & 0 & 1,00 & 1,14 & 1 & 0 & 0 & 0,33 & 0,46 \\
\hline \multicolumn{7}{|c|}{ Total } & 88 & 100 & & & & 72 & 100 \\
\hline
\end{tabular}


Tabla 2. ANOVA para el carácter ocupacional y los meses del estudio.

\begin{tabular}{|c|c|c|c|c|c|}
\hline Fuente & Suma de Cuadrados & Gl & Cuadrado Medio & Razón-F & Valor-P \\
\cline { 1 - 5 } Efectos principales & 266,13 & 2 & 133,06 & 16,12 & 0,00 \\
\hline A:Carácter & 17,71 & 5 & 3,54 & 0,43 & 0,82 \\
\hline B:Meses & 1320,73 & 160 & 8,25 & & \\
\hline Residuos & 1604,57 & 167 & & & \\
\hline Total & &
\end{tabular}

residentes continuas $(\mathrm{RC})$ de las otras dos categorías, lo que permite asumir la importancia del jagüey, en cuanto al mantenimiento de una comunidad residente permanente (Tabla 3). Al analizar estadísticamente mediante ANOVA, la variable individuos por especie presentes en el jagüey, independientemente del carácter y del tiempo, se obtiene que existe diferencia significativa entre especies (Tabla 4).

La diferencia hallada en cuanto al carácter ocupacional muestra la importancia del jagüey como refugio en un área desforestada, se le relaciona igualmente con una mayor abundancia de especies de aves, siendo las de mayor aporte dentro de estas áreas las especies residentes, concordando con Salinas et al. (2007), lo cual, es significativamente evidente en este estudio y se puede comprobar al observar en la tabla 1, la representación porcentual de las especies residentes continuas (RC), tales como: Jacana jacana (gallito de ciénaga), Crotophaga ani (cocinera), Pitangus sulphuratus (chichafria), Campylurhyncus griseus (chupa huevo), Bubulcus ibis (garza del ganado), Nycticorax nycticorax (guaco), Tigrisoma lineatum (vaco tigre).

En cuanto a la estructura de la comunidad y los diferentes tipos de ocupación específica mostrada y sin que el factor temporal se discrimine, este estudio difiere de diversos reportes, en donde la comunidad de aves presenta variaciones específicas de abundancia, durante los diferentes periodos estacionales del año, aún en el trópico. Indican, que se registran diferencias significativas en la abundancia y en el número de especies entre estaciones, lo que puede estar influenciado por el arribo de aves migratorias, la alta disponibilidad de alimento y la disponibilidad de sitios para criar (Isacch \& Martínez, 2001), algo que no se presentó en el área de estudio, en donde la avifauna estudiada es característicamente local, además por razones antrópicas, se localiza concentrada en lugares particulares, como los

Tabla 3. Análisis de diferencias mínimas significativas para el carácter: (RC) residente continuo, (VR) visitante regular, (VO) visitante ocasional. Según prueba de múltiples rangos (95\% LSD).

\begin{tabular}{|c|c|c|c|}
\hline Contraste & Sig. & Diferencia & +/- Límites \\
\hline $\mathrm{RC}-\mathrm{VO}$ & $*$ & 2,27 & 1,01 \\
\hline $\mathrm{RC}-\mathrm{VR}$ & $*$ & 2,89 & 1,12 \\
\hline $\mathrm{VO}-\mathrm{VR}$ & & 0,61 & 1,14 \\
\hline
\end{tabular}

* indica una diferencia significativa.

Tabla 4. ANOVA para individuos por especie $(\mathrm{p}<0.05)$.

\begin{tabular}{|c|c|c|c|c|c|}
\hline Fuente & Suma de Cuadrados & Gl & Cuadrado Medio & Razón-F & Valor-P \\
\hline Entre grupos & 1340,91 & 27 & 49,66 & 26,37 & 0,00 \\
\hline Intra grupos & 263,66 & 140 & 1,88 & 32,56 & 0,36 \\
\hline Total & 1604,57 & 167 & & & \\
\hline
\end{tabular}


cuerpos de agua remanentes. La composición específica de aves en diferentes ecosistemas depende de factores que actúan a nivel espacial y temporal (Hernández- Vásquez, 2000; Isacch \& Martínez, 2001).

Se señala la existencia de especies gregarias, que reúnen una fracción importante de su población en ciertos lugares formando colonias reproductivas o en sitios de descanso o de permanencia temporal o en zonas alimentación (BirdLife Internacional \& Conservation Internacional, 2005), como lo registran Salinas et al. (2007), para Ardea alba (Ciconiiformes, Ardeidae) y para Gallinula chloropus (Gruiformes, Rallidae); no obstante, el carácter gregario podría ser aplicado a las especies residentes permanentes o continuas, dadas las circunstancias de aislamiento y de ausencia de otros jagüeyes cercanos o de áreas aptas para su sobrevivencia, por ejemplo, Jacana jacana (Charadriiformes, Jacanidae).

Las 28 especies halladas en este estudio representan el $15,5 \%$ del total de la avifauna registrada como asociada a manglares y humedales del delta-estuario del río Magdalena, dentro de la región Caribe de Colombia, la cual, posee ecosistemas diversos y forma parte de la vía Parque Isla Salamanca y del Santuario de Fauna y Flora de la Ciénaga Grande de Santa Marta, con un total de 79.200ha (MorenoBejarano \& Álvarez-León, 2003).

De las especies de avifauna asociada a manglares y humedales del delta-estuario del río Magdalena, se registran como comunes Bubulcus ibis, Milvago chimachima, Jacana jacana, Aratinga pertinax, Crotophaga major, Pitangus sulphuratus, Progne tapera y Campylurhyncus griseus, lo que es consecuentemente similar para este estudio; sin embargo, Columbina talpacoti, Crotophaga ani, Tyrannus melancholicus y Thraupis episcopus, que fueron comunes en este trabajo, aparecen como escasas para la citada zona del Magdalena (Moreno-Bejarano \& Álvarez-León, 2003).

Hay que anotar que como visitantes regulares, las especies detectadas de las familias Cathartidae, Accipitridae y Falconidae, que agrupan en total seis especies dentro de esta categoría, representan el $85,7 \%$, esto es explicable por sus hábitos carroñeros o por ser aves de rapiña, lo que implica que el jagüey se convierte en un lugar de forrajeo importante para ellos en su búsqueda de alimento.

En cuanto a las aves rapaces, se ha demostrado que algunas especies de este grupo son favorecidas por los paisajes agrícolas, como Buteo magnirostris y Milvago chimachima, siendo está ultima la de mayor abundancia relativa, dentro de esta categoría en este trabajo, lo que permite aseverar que su presencia es favorecida por los paisajes ganaderos, tipificándose como abundante (Filloy \& Bellocq, 2007). Es claro que muchas especies de aves rapaces son tolerantes a los cambios ambientales que sufre una región en razón de la producción agropecuaria y de ocupación humana (Filloy \& Bellocq, 2007).

La pérdida de hábitats naturales es la principal causa de la pérdida de la biodiversidad mundial (Myers et al. 2000); no obstante, algunos agroecosistemas pueden conservar una sustancial parte de la biodiversidad de su anterior ecosistema natural y servir como zonas de amortiguación y complementarias de las áreas protegidas o de reserva para la fauna silvestre local o migratoria (Belfrage et al. 2005).

En este caso, los jagüeyes son sin duda alguna refugios de gran valor para la avifauna dentro de una matriz paisajística que cambió sustancialmente, debido a su dedicación agrícola, sin que pueda asumirse que la ganadería es la única responsable del cambio ambiental existente. Se le abona, en este caso, que permite comparativamente un mejor mantenimiento de la diversidad biológica, al menos en lo que a avifauna hace referencia (Fajardo et al. 2008; Botero et al. 2009). En muchos casos, el jagüey es la única reserva de agua disponible para el ganado y para los pobladores locales durante la época de sequía; es una fuente de sobrevivencia de gran valor cultural y productivo.

Conflicto de intereses: El manuscrito fue preparado y revisado con la participación de todos los autores, quienes declaramos que no existe ningún conflicto de intereses que ponga en riesgo la validez de los resultados presentados. Financiación: Este estudio fue financiado con aportes del Grupo de Investigación en Biodiversidad Tropical de la Universidad de Sucre, Colombia.

\section{BIBLIOGRAFÍA}

1. BELFRAGE, K.; BJÖRKLUND, J.; SALOMONSSON, L. 2005. The effects of farm size and organic farming on diversity of birds, pollinators, and plants in a Swedish Landscape. J. Hum. Behav. Soc. Environ. 34(8):582-588.

2. BIRDLIFE INTERNACIONAL.; CONSERVATION INTERNACIONAL. 2005. Áreas Importantes para la Conservación de las Aves en los Andes Tropicales: sitios prioritarios para la conservación de la biodiversidad. BirdLife and CI. 14:85-96.

3. BOTERO, A.L.; DE LA OSSA, V.J.; ESPITIA, P.A.; DE LA OSSA-LACAYO, A. 2009. Importancia de los jagüeyes en las sabanas del Caribe colombiano. Recia. 1(1):71-84. 
4. CANAVELLI, S.; ZACCAGNINI, M.E.; TORRESIN, J.; CALAMARI, N.; DUCOMMUN, M.; CAPLLONCH, P. 2004. Monitoreo extensivo de aves en el Centro-Sur de Entre Ríos. INSUGEO. 12:49-362.

5. DE LA OSSA, V.J.; SAMPEDRO-MARÍN, A.; GALVÁNGUEVARA, S.; DE LA OSSA-LACAYO, A. 2009. Registro de Pseudis paradoxa (Linnaeus, 1758) (Anura, Pseudidae) en el departamento de Sucre, Colombia. Recia. 1(2):264-272.

6. DECLERCK, S.; DE BIE, T.; ERCKEN, D.; HAMPEL, H.; SCHRIJVERS, S.; VAN WICHELEN, J. 2006. Ecological characteristics of small farmland ponds: Associations with land use practices at multiple spatial scales. Biol. Conserv. 131(4):523-532.

7. DÍAZ, I.A.; ARMESTO, J.J. 2003. La conservación de las aves silvestres en ambientes urbanos de Santiago. Rev Ambiente y Desarrollo de CIPMA. 19(2):31-38.

8. DUNN, E.H.; RALPH, C.J. 2004. The use of mist nets as a tool for bird population monitoring. Monitoring bird populations using mist nets. Studies in Avian Biology. 29:1-6.

9. FAJARDO, N.D.; GONZÁLEZ, J.R.; NEIRA, L.A. 2008. Ganadería del futuro. En: Fajardo, N.D.; González, J.R.; Neira, L.A. ed. Investigación para el desarrollo. Sistemas ganaderos amigos de las aves. CIPAV. p.173-225.

10. FARNSWORTH, G.L.; POLLOCK, K.H.; NICHOLS, J.D.; SIMONS, T.R.; HINES, J.E.; SAUER, J.R. 2002. A removal model for estimating detection probabilities from point-count surveys. Auk. 119:414-425.

11. FILLOY, J.; BELLOCQ, M.I. 2007. Respuesta de las aves rapaces al uso de la tierra: un enfoque regional. Hornero. 22(2):131-140.
12. HERNÁNDEZ-VÁSQUEZ, S. 2000. Aves acuáticas del estero de la Manzanilla, Jalisco, México. Acta Zool. Mex. 80:143-153.

13. ISACCH, J.P.; MARTÍNEZ, M.M. 2001. Estacionalidad y relaciones con la estructura del habitat de la comunidad de aves de pastizales de paja colorada (Paspalum quadrifarium) manejados con fuego en la provincia de Buenos Aires, Argentina. Ornitol. Neotrop. 12:345-354.

14. MAHECHA, L.; GALLEGO, L.A.; PELÁEZ, F.J. 2002. Situación actual de la ganadería de carne en Colombia y alternativas para impulsar su competitividad y sostenibilidad. Rev. Col. Cienc. Pec. 15(2):213-225.

15. MORENO-BEJARANO, L.M.; ÁLVAREZ-LEÓN, R. 2003. Fauna asociada a los manglares y otros humedales en el delta-estuario del río Magdalena, Colombia. Rev. Acad. Colomb. Cienc. 27(105):517-534.

16. MYERS, N.; MITTERMEIER, R.A.; MITTERMEIER, C.G.; DA FONSECA, G.A.B.; KENT, J. 2000. Biodiversity hotspots for conservation priorities. Nature. 403:853-858.

17. REMSEN, J.; CADENA, C.; JARAMILLO, A.; NORES, M.; PACHECO, J.; ROBBINS, M.T. 2008. A classification of the bird species of South America. AOU:10-65.

18. SÁENZ, J.C.; MENACHO, O.R.M. 2005. Riqueza y abundancia de las aves migratorias en paisajes agropecuarios de Esparza, Costa Rica. Zeledonia. 9(1):10-21.

19. SALINAS, L.; ARANA, C.; PULIDO, V. 2007. Diversidad, abundancia y conservación de aves en un agroecosistema del desierto de Ica, Perú. Rev Perú Biol. 13(3):155-167.

Recibido: Noviembre 23 de 2011

Aceptado: Abril 22 de 2012 